
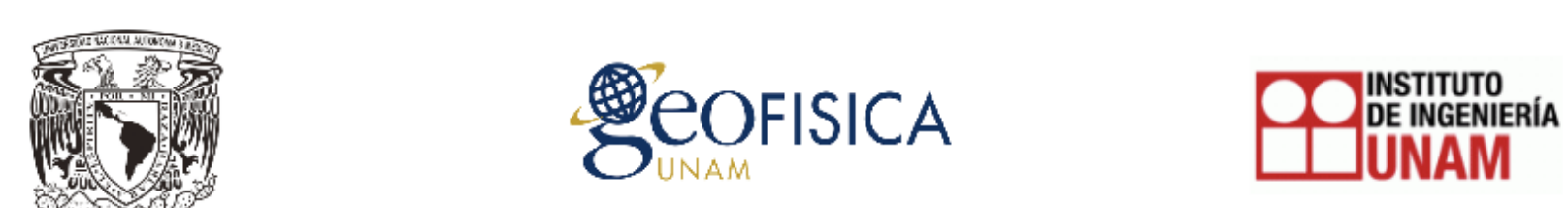

\title{
¿Qué ocurrió el 19 de septiembre de 2017 en México?
}

\author{
Grupos de Sismología e Ingeniería de la UNAM \\ Dr. Víctor Manuel Cruz Atienza \\ Departamento de Sismología, Instituto de Geofísica, \\ Universidad Nacional Autónoma de México (UNAM)
}

Dr. Shri Krishna Singh

Sismólogo y Profesor Emérito, Instituto de Geofísica, UNAM

Dr. Mario Ordaz Schroeder

Coordinación de Ingeniería Sismológica, Instituto de Ingeniería, UNAM

Nota: La información utilizada para elaborar esta nota resulta del esfuerzo de investigadores y técnicos académicos de los institutos de Geofísica e Ingeniería de la UNAM.

Mucho nos preguntamos si el sismo, de magnitud 7.1, fue más fuerte en la Ciudad de México que el terremoto de magnitud 8.0 de 1985. Sólo por la enorme diferencia en magnitud de los dos eventos, uno podría suponer que no. Esto tiene sentido, ya que el sismo de 1985 liberó 32 veces más energía sísmica que el del 19 de septiembre de 2017. Sin embargo, en 1985, el epicentro fue muy lejano y bajo las costas del estado de Michoacán, a más de 400 km de la capital, mientras que el 7.1 ocurrió apenas $120 \mathrm{~km}$ al sur de la ciudad. Al propagarse, las ondas sísmicas se atenúan rápidamente. Por ello, a pesar de que la ruptura que generó las ondas sísmicas el martes pasado es mucho menor que la de 1985, las sacudidas en la Ciudad de México fueron tan violentas. A continuación, veremos porqué.

\section{¿Dónde y por qué ocurrió el sismo?}

La ruptura del sismo del 19 de septiembre de 2017 ocurrió dentro de la placa oceánica de Cocos (i.e. sismo intraplaca), por debajo del continente, a una profundidad de 57 km (Figura 1). Si bien este tipo de sismo no es el más común en México, de ninguna manera es extraordinario. En la Figura 1 se muestran los epicentros y profundidades de algunos sismos similares, incluyendo el del pasado martes. Estas rupturas se producen a profundidades mayores que los típicos sismos de subducción como el de 1985, que tiene lugar bajo las costas del Pacífico mexicano sobre la interfaz de contacto entre las placas tectónicas de Cocos y de Norteamérica (línea roja, Figura 1). Los sismos intraplaca, de profundidad intermedia, se producen por esfuerzos extensivos a lo largo de la placa de Cocos. Las fallas geológicas asociadas a estos sismos se conoces con el nombre de "fallas normales". Es preciso mencionar que estudios realizados para sismos intraplaca en México muestran que, por año, la probabilidad de que la intensidad de las sacudidas en la Ciudad de México debidas a este tipo de terremotos sea grande es muy similar a la de los sismos típicos de subducción, como el de 1985, entre otros. Esto implica que el peligro sísmico en la capital, asociado a los sismos intraplaca (como los del 7 y 19 de septiembre de 2017), es tan 
Figura 1. Localizaciones del sismo de magnitud 7.1 del 19 de septiembre de 2017 (color rojo) y algunos otros del mismo tipo en la región. Las "pelotas de playa" ilustran la orientación de las fallas y la dirección en que deslizaron. Todas estas son fallas de tipo normal. grande como el de los sismos más comunes que ocurren bajo las costas del Pacífico mexicano.

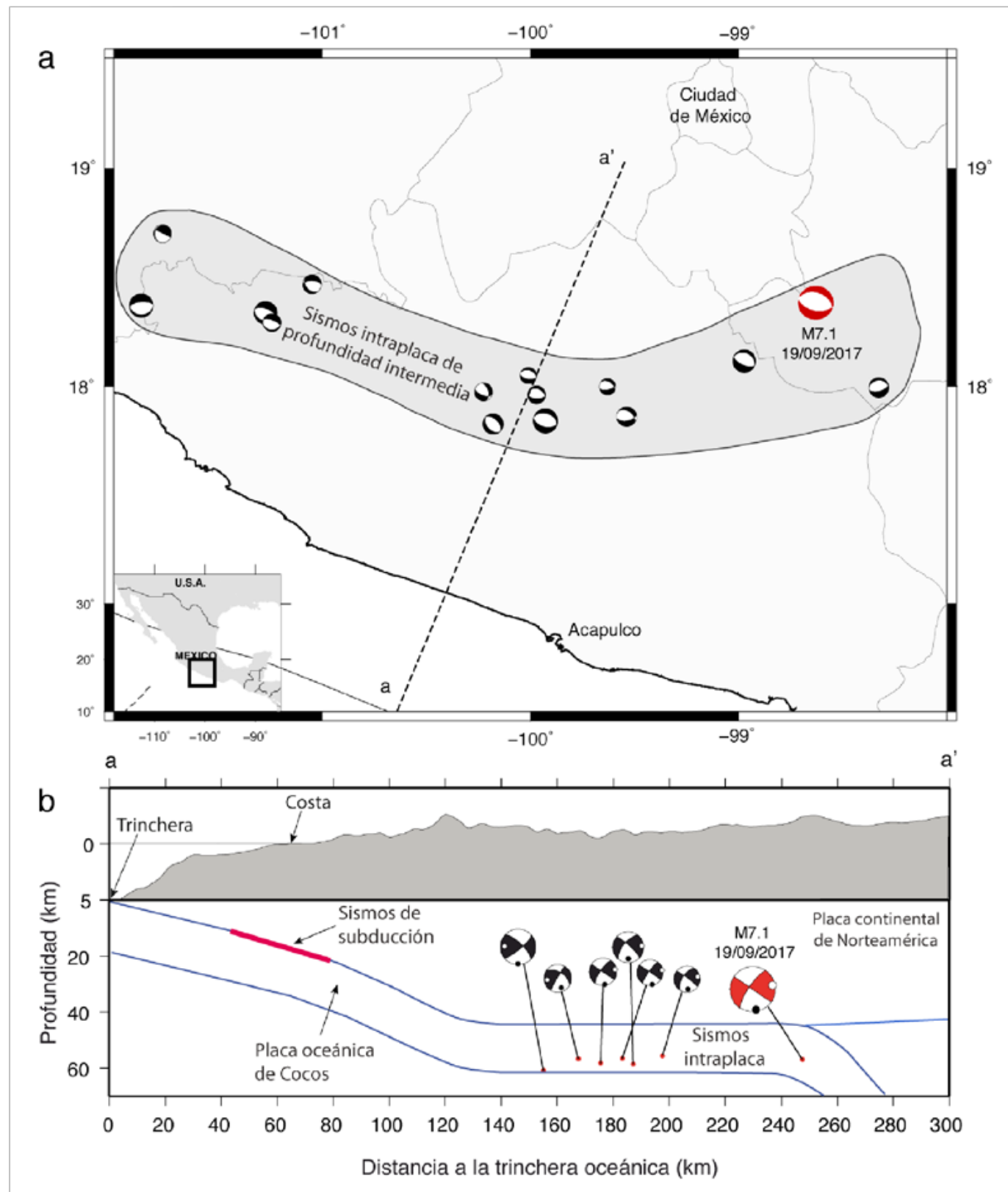

\section{¿Por qué tantos daños?}

Gracias a la vasta red de acelerógrafos y sismómetros que registraron ambos terremotos en la Ciudad de México, y a los esfuerzos de muchos sismólogos e ingenieros mexicanos, hoy hemos entendido mejor qué ocurrió. Uno de los ingredientes que usan los ingenieros civiles para calcular las estructuras de los 
Figura 2. Espesor de la cuenca sedimentaria donde se encuentra gran parte de la Ciudad de México. Nótese la localización del terremoto del 19 de septiembre en el cuadro de la parte superior izquierda. Los puntos azules indican los sitios de dos estaciones sísmicas que registraron los terremotos de 1985 y 2017. La región entre los contornos azul y rojo representa la zona de transición entre el suelo firme y el suelo blando. edificios de la CDMX es la aceleración máxima (Amax) del suelo producida por las ondas sísmicas. En 1985, la Amax en Ciudad Universitaria (CU), que está en suelo firme (Figura 2), fue de $30 \mathrm{gal}$ ( $1 \mathrm{gal}=1 \mathrm{~cm} / \mathrm{s}^{2}$ ), mientras que la Amax del 19 de septiembre de 2017 fue de 57 gal. Es decir que el suelo en la zona cercana a CU experimentó una sacudida dos veces mayor que en 1985.

Sin embargo, todos sabemos que gran parte de la Ciudad de México está edificada sobre sedimentos blandos de los antiguos lagos que existieron en el valle. Estos sedimentos provocan una enorme amplificación de las ondas sísmicas en la Ciudad de México que, probablemente, sea la más grande reportada en el mundo.

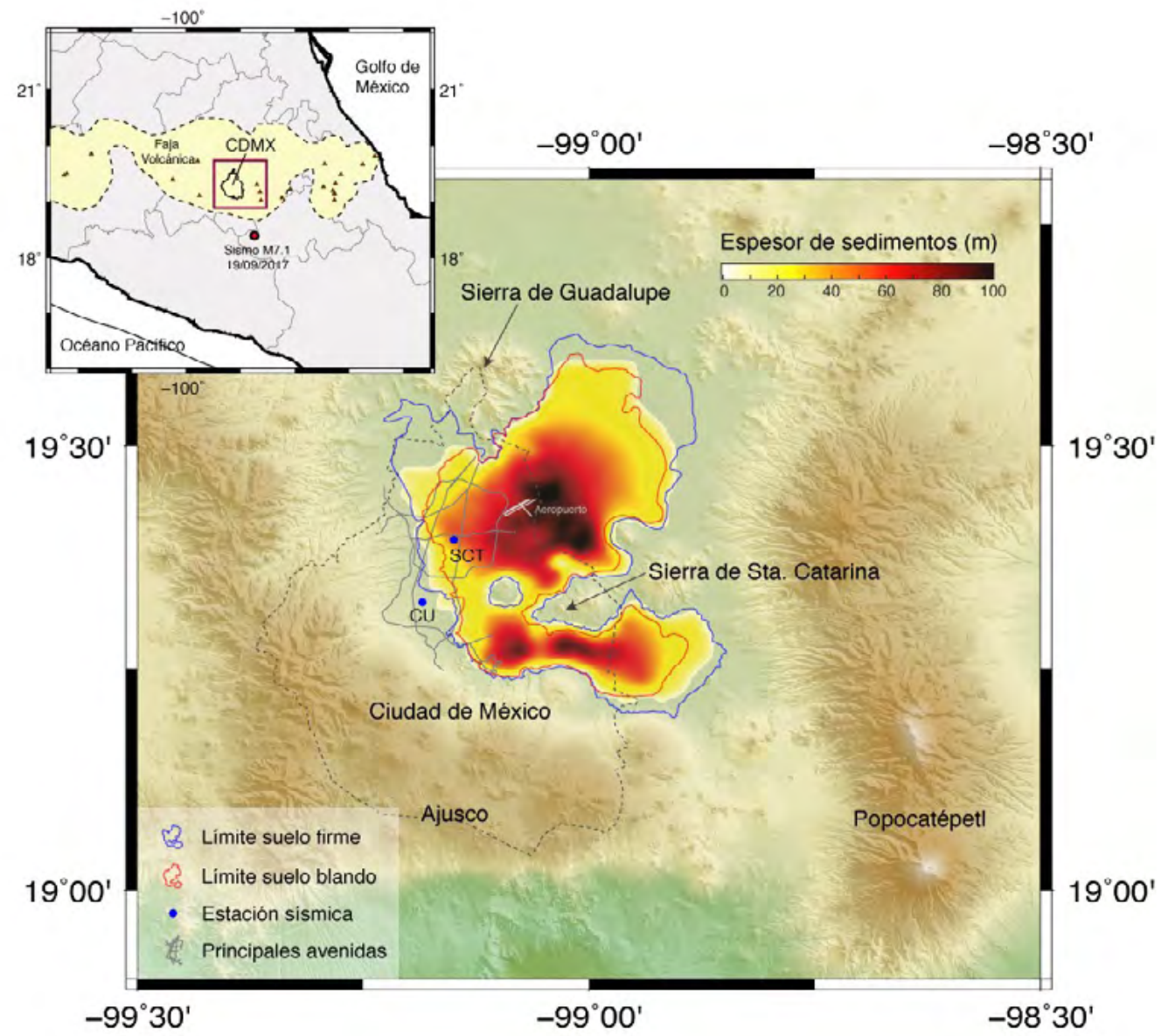

Para dar una idea tangible, la amplitud de las ondas sísmicas con períodos cercanos de 2 segundos en zona de lago (o zona blanda) (e.g. colonias Roma, Condesa, Centro y Doctores) puede llegar a ser 50 veces mayor que en un sitio de suelo firme de la Ciudad de México. Sin embargo, como las ondas también se amplifican en el suelo firme de la periferia, con respecto a lugares lejanos de la Ciudad de México, la amplitud en zona de lago puede ser de 300 a 500 veces mayor. En algunos sitios de la zona del lago, las aceleraciones máximas del suelo 
Figura 3.

Localización de daños graves y colapsos durante el sismo del 19 de septiembre de 2017 (puntos rojos). El mapa contiene de fondo la información del periodo natural del suelo (degradado de colores), que es una característica que determina el potencial de amplificación del suelo blando de la ciudad. La zona en tonos grises representa los periodos de 0.5 a 1.0 segundos, también conocida como la zona de transición. (Fuente: ERN Ingenieros Consultores, ERNTérate, "Nota de interés al respecto del sismo del 19 de septiembre de 2017", publicada el 23 de septiembre de 2017). producidas por el sismo de magnitud 7.1 fueron menores a las registradas en 1985. Por ejemplo, en la Secretaría de Comunicaciones y Transportes (SCT, Figura 2), que se encuentra en dicha zona, Amax en 1985 fue de 160 gal, mientras que el pasado 19 de septiembre fue de 91 gal. En otros sitios de la zona de lago, las aceleraciones del suelo durante el sismo reciente fueron, muy probablemente, mayores que la registradas en 1985. Se trata de un patrón de movimiento complejo y muy variable en el espacio.

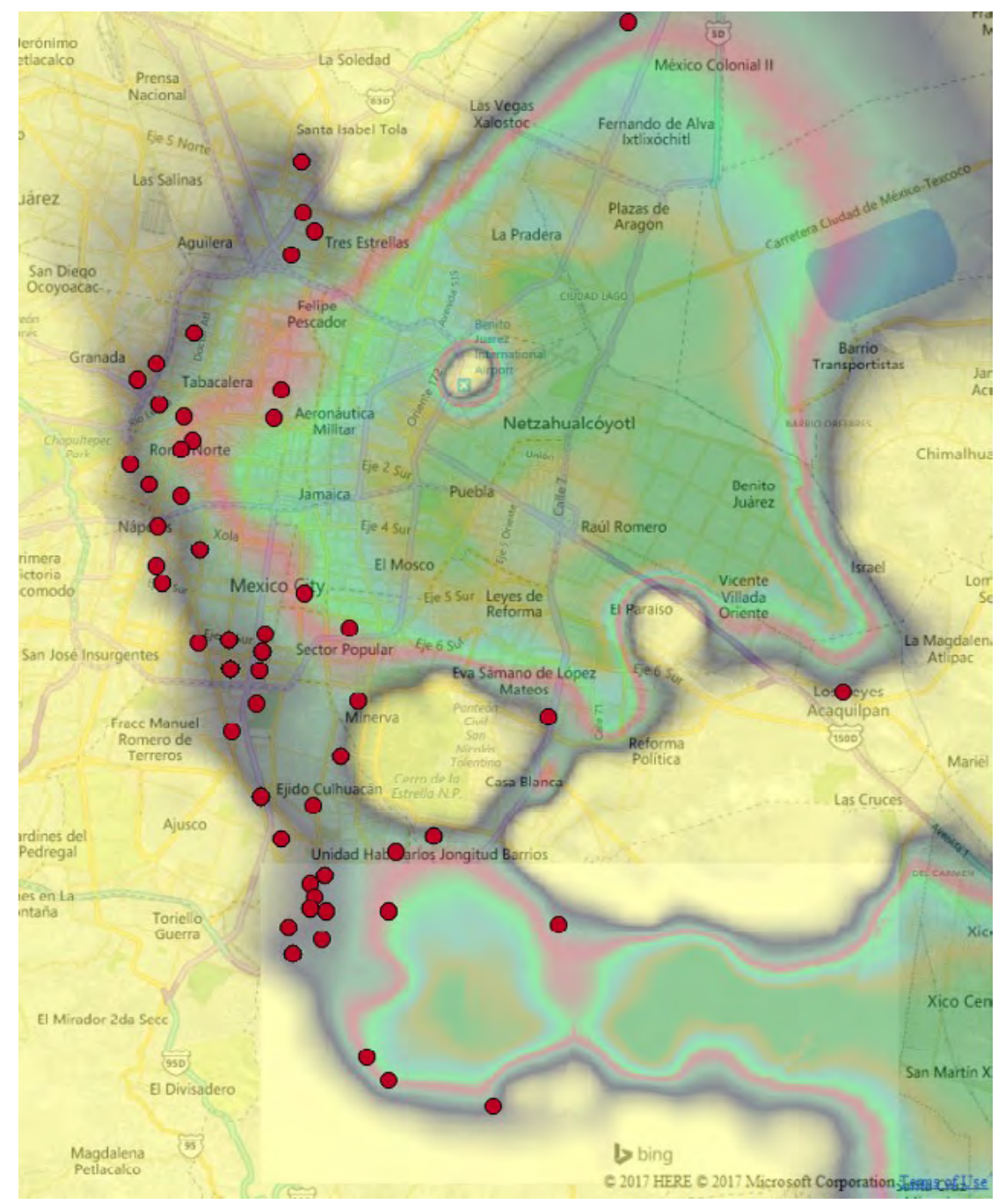

Un análisis detallado del movimiento del suelo producido por ambos sismos en la Ciudad de México revela cosas interesantes. De la misma manera que sucede con el sonido emitido por una cuerda de guitarra, los sismos están formados por ondas con diferentes períodos de oscilación. Los sismogramas registrados muestran que la amplitud de las ondas sísmicas con períodos de oscilación menores a 2 segundos fue mucho más grande en 2017 que en 1985 (en promedio unas 5 veces), grosso modo, en toda la ciudad. Sorprendentemente, sucede lo contrario para ondas con períodos mayores de 2 segundos, 
cuya amplitud fue mucho mayor en 1985 (hasta 10 veces mayor). Como veremos abajo, esto tiene fuertes implicaciones en el tipo de daños observados durante ambos terremotos.

En resumen, los movimientos del suelo debidos al sismo de magnitud 7.1 fueron muy violentos y, de cierto modo, comparables a los de 1985 a pesar de haber sido provocados por una ruptura (falla geológica) mucho más pequeña que, sin embargo, ocurrió mucho más cerca de la Ciudad.

\section{Y los edificios, ¿qué sintieron?}

Para los edificios, la situación no es tan sencilla. La aceleración máxima del suelo (Amax) no es necesariamente lo que pone en riesgo su estabilidad. Por el contrario, al ser estructuras de dimensiones (alturas) diferentes, su vulnerabilidad es muy variada. Ondas con mayor período de oscilación amenazan estructuras más altas. Contrariamente, ondas con períodos más cortos, amenazan estructuras más bajas. Para identificar qué estructuras pudieron verse afectadas por el sismo de 2017, los ingenieros y sismólogos calculan lo que llaman las "aceleraciones espectrales" a partir de los sismogramas registrados. Dichos valores nos dan una idea de las aceleraciones que pudieron experimentar, en sus azoteas, edificios con diferentes alturas. Las aceleraciones espectrales en CU (suelo firme) indican que, los edificios de 1 a 12 pisos cercanos a la estación sísmica experimentaron una aceleración promedio de 119 gal, que es aproximadamente 2 veces mayor que la observada en 1985 (Figura 4a). En contraste, las estimaciones en SCT (suelo blando) muestran que edificios pequeños de este tipo, cercanos a la estación, experimentaron una aceleración promedio de 188 gal, muy similares a las de 1985 (Figura 4b).

Por otro lado, edificios más altos, de entre 12 y 20 pisos, experimentaron una aceleración promedio en CU de 60 gal, que es 30\% menor a la de 1985, que fue de 85 gal (Figura 4a). La diferencia más clara entre los dos terremotos ocurrió en suelo blando para edificios con más de 15 pisos. La Figura 4b muestra claramente cómo, en 1985, los edificios de este tipo cercanos a SCT experimentaron aceleraciones de 1.5 a 4.9 veces más grandes que las observadas el 19 de septiembre de 2017. En 1985, algunas de estas grandes estructuras experimentaron aceleraciones de hasta 760 gal. Como referencia, la aceleración de la gravedad terrestre (i.e. la de un cuerpo en caída libre) es de 981 gal.

Como veremos a continuación, la estación SCT no se encuentra en la zona con los mayores daños, que se encuentra más al oeste (hacia las colonias Roma y Condesa), principalmente en la zona de transición de la cuenca sedimentaria. Un análisis similar al de la Figura 4 a partir de registros en dichas colonias permitirá estimar qué tipos de edificios fueron los más amenazados. En esa zona, esperamos aceleraciones mayores que las de SCT para edificios de 4 a 10 pisos. 
Figura 4.

Aceleraciones experimentadas en las azoteas de edificios con diferentes alturas en los sitios

CU (a, suelo firme) y SCT (b, suelo blando) (ver Figura 2) para los sismos del 19 de septiembre de 1985 (rojo) y 2017 (azul).

$1 \mathrm{gal}=1 \mathrm{~cm} / \mathrm{s}^{2}$. Las aceleraciones reportadas corresponden al promedio geométrico de ambas componentes horizontales del movimiento.

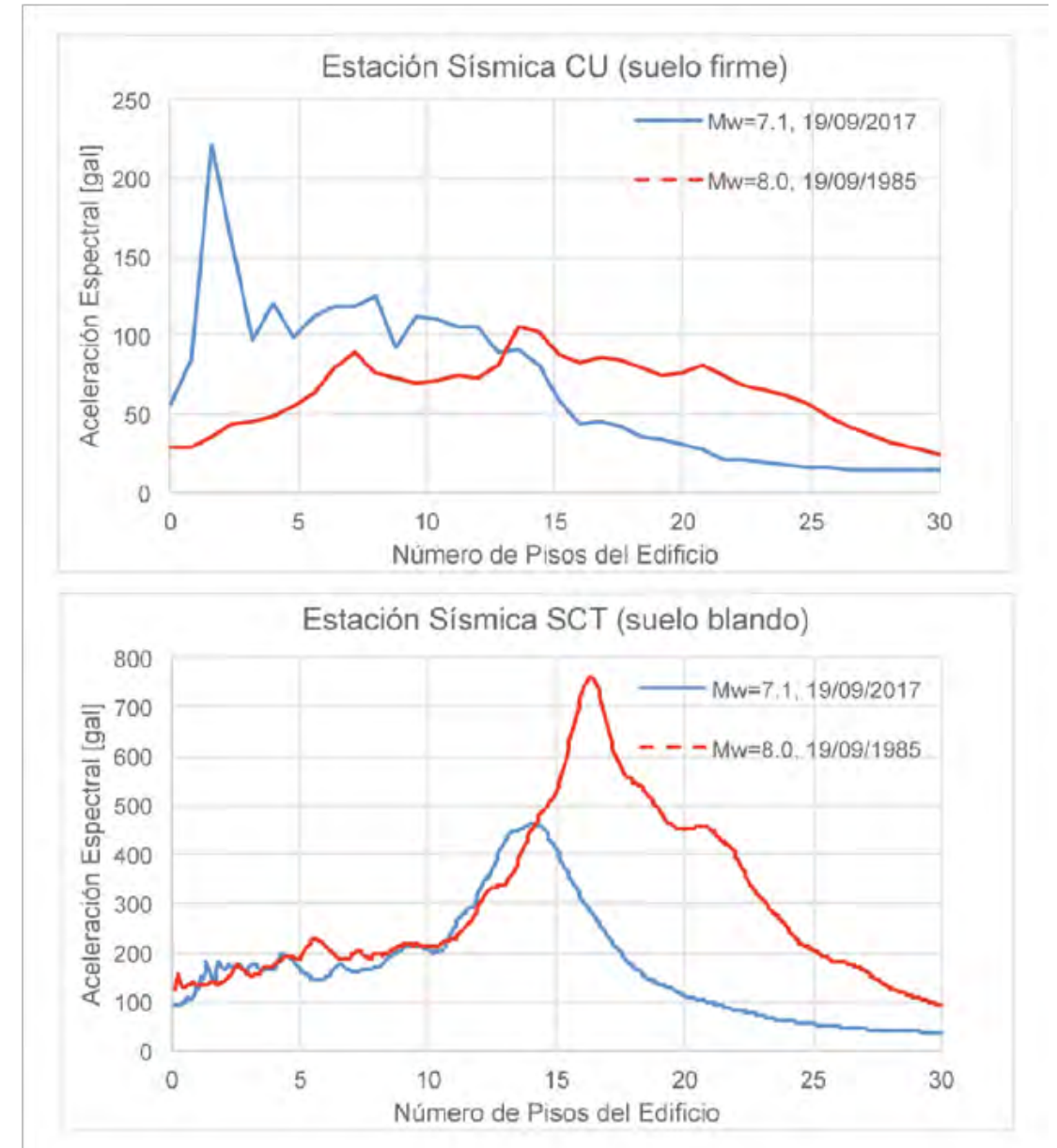

Los ingenieros y sismólogos de la UNAM, gracias a múltiples investigaciones basadas en miles de registros sísmicos en la Ciudad de México y el desarrollo de herramientas sofisticadas han podido cartografiar, en toda la mancha urbana, valores de aceleración experimentados el pasado 19 de septiembre para diferentes tipos de estructuras. Dichas herramientas fueron desarrolladas en el Instituto de Ingeniería de la UNAM y operan automáticamente en tiempo real. Con ellas, se generan mapas de intensidad en toda la ciudad pocos minutos después del sismo, mismos que son útiles para identificar, rápidamente, las zonas potencialmente dañas. La Figura 5 ilustra claramente esto para el sismo del 19 de septiembre de 2017. Ahí se puede apreciar que existe una clara correlación entre los daños ocurridos (i.e. los edificios colapsados o fuertemente dañados) y las zonas donde se produjeron las mayores aceleraciones espectrales. Consistentemente con lo explicado en el párrafo anterior, el sismo de magnitud 7.1 dañó, en su mayor parte, estructuras relativamente pequeñas, de entre 4 y 7 pisos, a lo largo de una franja con orientación norte-sur dentro de la zona de transición (entre las zonas de suelo firme y blando) al poniente de la zona de lago (Figuras 3 y 4). En contraste, las estructuras dañadas en 1985 fueron en su mayoría más grandes, con alturas de entre 7 y 14 pisos. 


\section{Figura 5.}

Mapa de aceleraciones espectrales para periodos de 1 segundo, correspondientes a la respuesta de estructuras de 7 a 10 pisos. Los triángulos negros muestran las localizaciones de los edificios colapsados o fuertemente dañados.

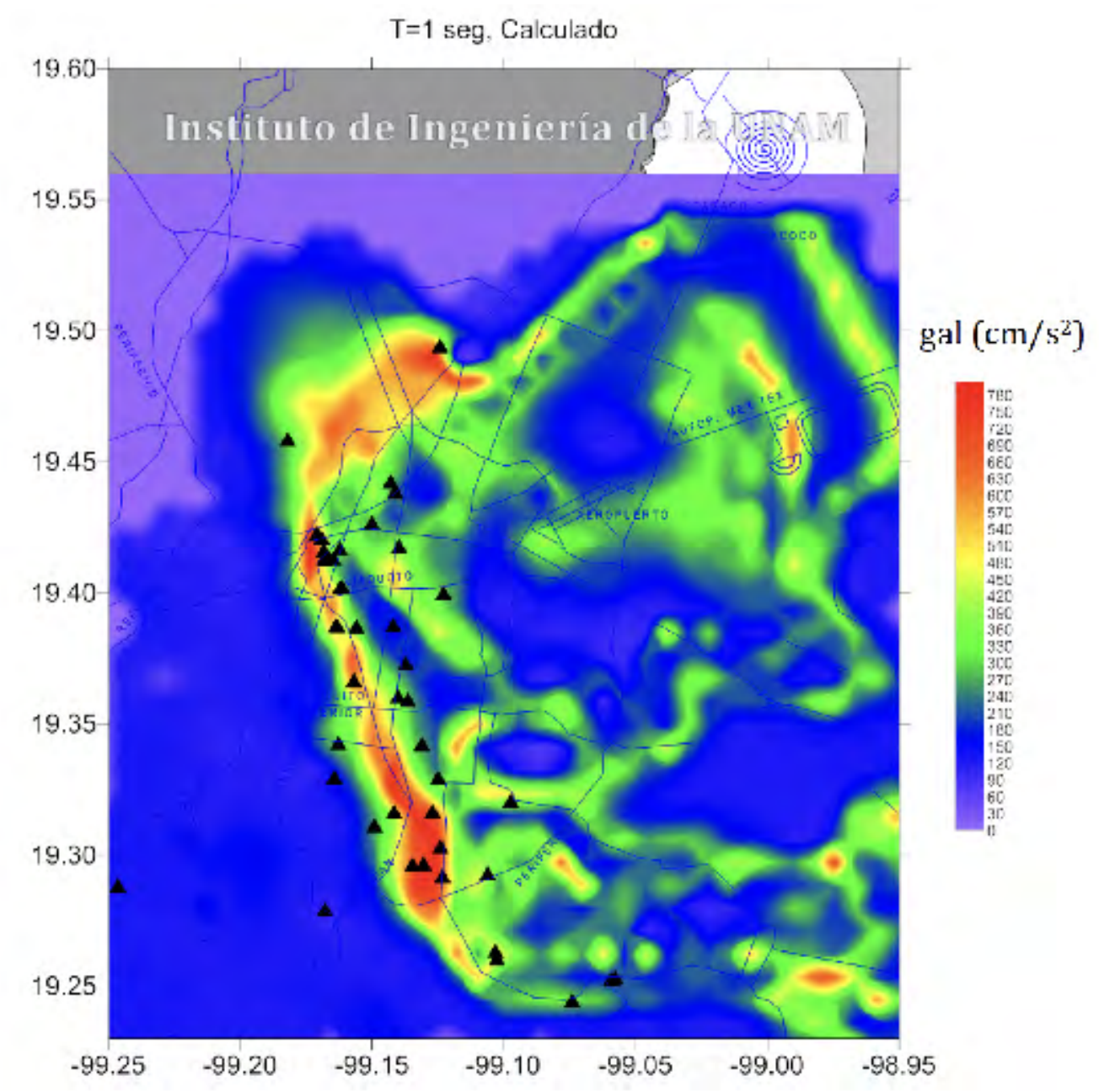

\section{¿Por qué los daños se concentraron en ciertas zonas de la ciudad?}

La violencia del movimiento del suelo en la Ciudad de México depende principalmente del tipo de suelo donde nos encontremos. Como ya se dijo, gran parte de la ciudad está asentada en suelo blando, sobre sedimentos lacustres (contorno rojo en de Figura 1). La Figura 5 muestra la aceleración estimada en las azoteas de edificios de 7 a 10 pisos (i.e. con períodos de resonancia cercanos a 1 segundo) provocada por el sismo del 19 de septiembre de 2017. Cabe precisar que este mapa fue generado en forma automática, casi en tiempo real, por el Instituto de Ingeniería de la UNAM, por lo que se hizo público unos minutos después del sismo. Como ya se dijo, existe una clara correlación entre la franja roja de máxima aceleración al poniente de la cuenca y la localización de los edificios colapsados o fuertemente dañados. También es sorprendente la correlación que hay entre los valores grandes de aceleración (franja roja) y la geometría (espe- 
sor) de los sedimentos lacustres (Figuras 2 y 3). La mayoría de los daños se encuentran al oeste de la cuenca sedimentaria, sobre la zona de transición y parte del suelo blando, muy cerca de su límite poniente. Ahí, los sedimentos tienen un espesor de 10 a $30 \mathrm{~m}$. La interacción y amplificación de las ondas sísmicas con esta región de la cuenca sedimentaria provocaron los daños.

Además de la amplificación de las ondas, la duración del movimiento del suelo es también mucho mayor dentro de los sedimentos blandos. Estudios recientes muestran que las duraciones más grandes esperadas para períodos de oscilación menores a 2 segundos coinciden con la zona de mayor destrucción para el sismo de magnitud 7.1 del 19 de septiembre de 2017. Por ejemplo, la duración de la fase intensa del movimiento en CU fue de 36 segundos, mientras que en SCT, fue de 1 minuto. Por esta razón, tanto la violencia de las sacudidas como su duración en la zona de transición y de lago son las causantes de la destrucción.

\section{¿Los daños se debieron a deficiencias en el reglamento de construcción?}

No tenemos hasta el momento indicios de que las fuerzas de diseño (i.e. los criterios de resistencia estructural) actualmente vigentes en el reglamento de construcción de la Ciudad de México se hayan excedido durante el sismo del 19 de septiembre de 2017. Por lo tanto, los edificios construidos en los últimos años no deberían haber sufrido daños. Sin embargo, en el caso de estructuras comunes, el Reglamento de Construcciones de la ciudad no exige que las edificaciones antiguas sean reforzadas para resistir las fuerzas especificadas en las normas emitidas después de su fecha de construcción. Es posible, entonces, que en el caso de edificaciones antiguas sí se hayan excedido las fuerzas de diseño con las que fueron proyectadas.

Independientemente de lo anterior, se sabe que existe un grave problema por falta de cumplimiento de las normas especificadas en el reglamento vigente de construcción, documentado en proyectos de investigación realizados en la UNAM. En consecuencia, los daños observados se explican mejor con la falta de observancia de las normas, más que por posibles deficiencias en el Reglamento de Construcción actual.

\section{¿Esperamos un sismo de mayor intensidad en la Ciudad de México?}

Es muy probable. Bajo las costas del estado de Guerrero, por ejemplo, existe una brecha sísmica (i.e. segmento donde no ha ocurrido un terremoto significativo en más de 60 años) de 250 km de longitud en dónde podría ocurrir un 
sismo de magnitud superior a 8. Este segmento se encuentra a unos $300 \mathrm{~km}$ de la Ciudad de México. Es decir, aproximadamente $150 \mathrm{~km}$ más cerca que la zona epicentral del terremoto de 1985. Estimaciones hechas por sismólogos de la UNAM sugieren que, si este sismo ocurriera en un futuro, las aceleraciones del suelo blando en la Ciudad de México podrían ser, bajo ciertas condiciones, mayores que las del sismo reciente de magnitud 7.1, y de 2 a 3 veces mayores que las de 1985 en particular para edificios de más de 10 pisos. La duración del movimiento del suelo sería mayor que las experimentadas en 2017 (alrededor de 3 minutos en su fase intensa).

23 de septiembre de 2017

\section{Cómo citar este artículo}

- Cruz Atienza, Víctor Manuel, Singh, Shri Krishna y Ordaz Schroeder, Mario (2017). ¿Qué ocurrió el 19 de septiembre de 2017 en México?, en Revista Digital Universitaria (RDU), vol. 18, núm. 7, septiembre-octubre. Recuperado de http://dx.doi. org/10.22201/codeic.16076079e.2017.v18n7.a10 